\title{
Measurement of spontaneous emission from a two-dimensional photonic band gap defined microcavity at near-infrared wavelengths
}

\author{
R. K. Lee, ${ }^{\text {a) }}$ O. J. Painter, B. D'Urso, A. Scherer, and A. Yariv \\ Applied Physics and Electrical Engineering, California Institute of Technology, 1200 E. California \\ Boulevard, Pasadena, California 91125
}

(Received 8 September 1998; accepted for publication 18 January 1999)

\begin{abstract}
An active, photonic band gap-based microcavity emitter in the near infrared is demonstrated. We present direct measurement of the spontaneous emission power and spectrum from a microcavity formed using a two-dimensional photonic band gap structure in a half wavelength thick slab waveguide. The appearance of cavity resonance peaks in the spectrum correspond to the photonic band gap energy. For detuned band gaps, no resonances are observed. For devices with correctly tuned band gaps, a two-time enhancement of the extraction efficiency was demonstrated compared to detuned band gaps and unpatterned material. (c) 1999 American Institute of Physics.
\end{abstract}

[S0003-6951(99)01611-3]

Photonic crystals have been predicted to be able to fundamentally modify spontaneous emission properties of light emitting materials. ${ }^{1,2}$ High $Q$ optical cavities are considered to be useful for high efficiency, low power and high modulation speed light sources. One dimensional photonic structures have been used successfully in light emitting diodes (LEDs) to enhance efficiency and to enhance spontaneous emission. ${ }^{3}$ Recently, two-dimensional (2D) structures have attracted a great deal of attention ${ }^{4-6}$ due to the simpler geometry, in terms of both fabrication complexity and device design as compared to three-dimensional structures. Such structures have been considered as a means of controlling in-plane spontaneous emission, a significant loss mechanism in vertical-emitting structures. ${ }^{4}$ However, experimental evidence $^{5,7}$ has indicated that finite hole depth and waveguide geometry can lead to strong scattering of light into the substrate which would limit the confinement possible with a 2D photonic crystal due to scattering out of the photonic crystal plane. Passive reflection, diffraction and transmission measurements have recently been carried out in twodimensional photonic crystal structures. ${ }^{8}$ Martorell and Lawandy, ${ }^{9}$ and subsequently Petrov et al. ${ }^{10}$ have observed inhibited spontaneous emission from low-index contrast three-dimensional periodic structures $\left(\epsilon_{r}=1.45\right)$. Berggren et al. ${ }^{11}$ have also made active devices by including a low index contrast two-dimensional periodicity. However, a complete band gap in two or three dimensions requires a relatively strong index of refraction contrast $\left(\epsilon_{r}>10\right) .{ }^{12}$

We have designed and fabricated two-dimensional photonic crystals in a thin semiconductor membrane bounded above and below by air. The optical cavity consists of a $\lambda / 2$ thick dielectric slab waveguide suspended in air. The strong confinement provided by the index contrast between the semiconductor membrane and air helps to reduce the scattering out of the plane of the waveguide by the photonic crystal. The membrane is patterned with a hexagonal twodimensional array of air holes. A number of holes can be omitted to form cavities of different shapes, providing in-

${ }^{a)}$ Electronic mail: leereg@cco.caltech.edu plane localization. The calculated band structure for this photonic crystal, taking into account the finite third dimension shows a TE band gap from $a / \lambda=0.276$ to $a / \lambda=0.362$. Details of this calculation have been previously described. ${ }^{4,13,14}$ The cavities were designed with the photonic band gap centered around the peak emission wavelength at a normalized frequency of approximately $a / \lambda=0.32$ (lattice spacing of $a$ $=500 \mathrm{~nm}$ for $\lambda=1550 \mathrm{~nm}$ ). In this letter, cavities approximately $15 \mu \mathrm{m}$ across were examined. At the emission wavelength of $1.55 \mu \mathrm{m}$ this structure forms a multimode cavity. A scanning electron micrograph of a typical device is shown in Fig. 1(a). A variety of devices were fabricated with lattice spacings from $300 \mathrm{~nm}$ to $1 \mu \mathrm{m}$ while maintaining $\mathrm{r} / \mathrm{a}$ $\approx 0.31$, thereby lithographically tuning the band gap ${ }^{15}$ across the emission spectrum. The membrane consists of $6 \mathrm{InGaAs}$ quantum wells with InGaAsP barriers grown by organometallic vapor phase epitaxy (OMVPE) on an InP substrate. The total waveguide thickness was $150 \mathrm{~nm}$. The InGaAsP system was chosen due to its relatively low surface recombination velocity. Fabrication of these devices has been previously described. ${ }^{16}$ These devices are, in essence, microdisk lasers ${ }^{17}$ bounded by PBG material so that the total internal reflection of the microdisk is replaced by Bragg reflection from the photonic crystal. A finite difference time domain calculation $^{13}$ of the resonant mode in this structure [Fig. $1(b)]$ is reminiscent of a microdisk whispering gallery mode even though the cavity is hexagonal rather than circular.

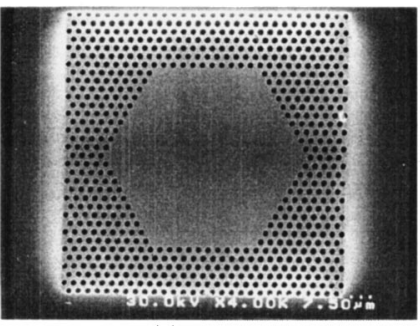

(a)

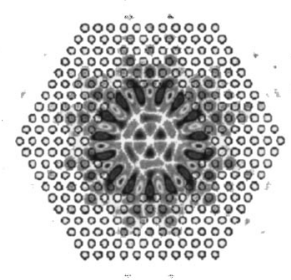

(b)
FIG. 1. (a) Scanning electron micrograph (top view) of a hexagonal membrane cavity bounded by 2D photonic band gap crystal. (b) Calculated field distribution. 


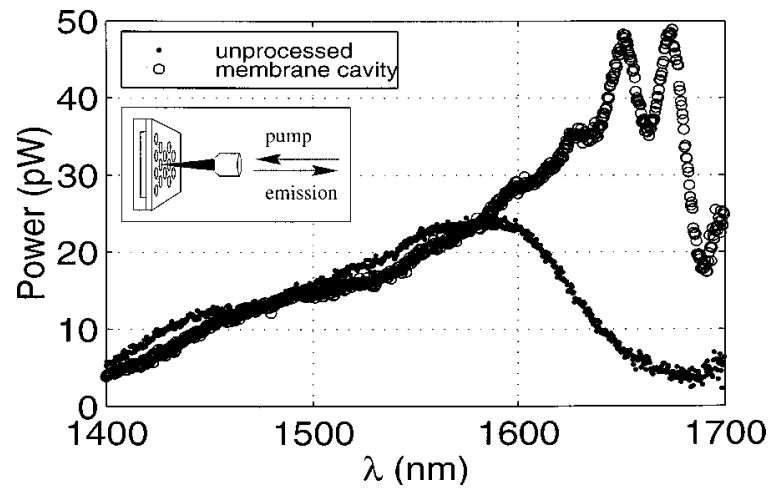

FIG. 2. Spectrum showing enhanced emission from hexagonal membrane cavity. Peak power from the membrane device shows a $2.0 \times$ enhancement. Pumping configuration schematic is shown in the inset.

Other types of modes also exist in this structure in addition to the whispering gallery-like modes. These are primarily modes which reflect between opposing faces of the hexagon. In these devices, it is possible to design the cavity modes by engineering the photonic band gap energy and by controlling the cavity shape.

The membrane microcavity was optically pumped by $200 \mathrm{nsec}$ pulses from a semiconductor laser $(\lambda=980 \mathrm{~nm})$ focused to a spot size of approximately $8 \mu \mathrm{m}$. Photoluminescence from the quantum wells at $\lambda \simeq 1.55 \mu \mathrm{m}$ was collected normal to the membrane (Fig. 2 inset). Figure 2 shows the spectrum from unpatterned material and from a cavity showing strong resonances superimposed on the broader quantum well emission. The emission peak from the hexagonal cavity has been redshifted approximately $70 \mathrm{~nm}$ due to heating of the membrane by the pump light, corresponding to a membrane temperature of $\approx 375 \mathrm{~K} .{ }^{18}$ The emission spectrum is significantly broadened and redshifted due to the high peak pumping density $\left(\sim 10 \mathrm{~kW} / \mathrm{cm}^{2}\right)$ as well as the poor thermal conduction path resulting from the membrane being suspended in air. All devices were fabricated in close proximity on the same wafer in order to minimize temperature variations in the measurements. The emission redshift was used to estimate the active layer temperature. Negligible differences were observed between devices at a given pumping level. This is an important consideration since heating can account for significant shift in the refractive index as well as change the spontaneous emission efficiency of the active layer material. The peak emission power from the hexagonal cavity has been enhanced by approximately $2.0 \times$ as compared to the unpatterned material as shown in Fig. 2. This enhancement is largely due to the fact that these structures are undercut, leaving an air gap beneath the membrane. This agrees quite well with a predicted normal direction emission power enhancement of $1.81 \times$ for dipole emission in this slab waveguide structure suspended in air as compared to the same waveguide on an InP substrate even though this measurement only gives the enhancement into the collection cone defined by the optics (numerical aperture $N A=0.6$ ). This spontaneous emission enhancement calculation has been previously described. ${ }^{19}$

The emission spectra from various hexagonal membrane cavities is shown in Fig. 3. Figure 3(a) corresponds to a relatively large lattice spacing of $a=760 \mathrm{~nm}$ and is very Downloaded 18 Dec 2005 to 131.215.240.9. Redistribution subject

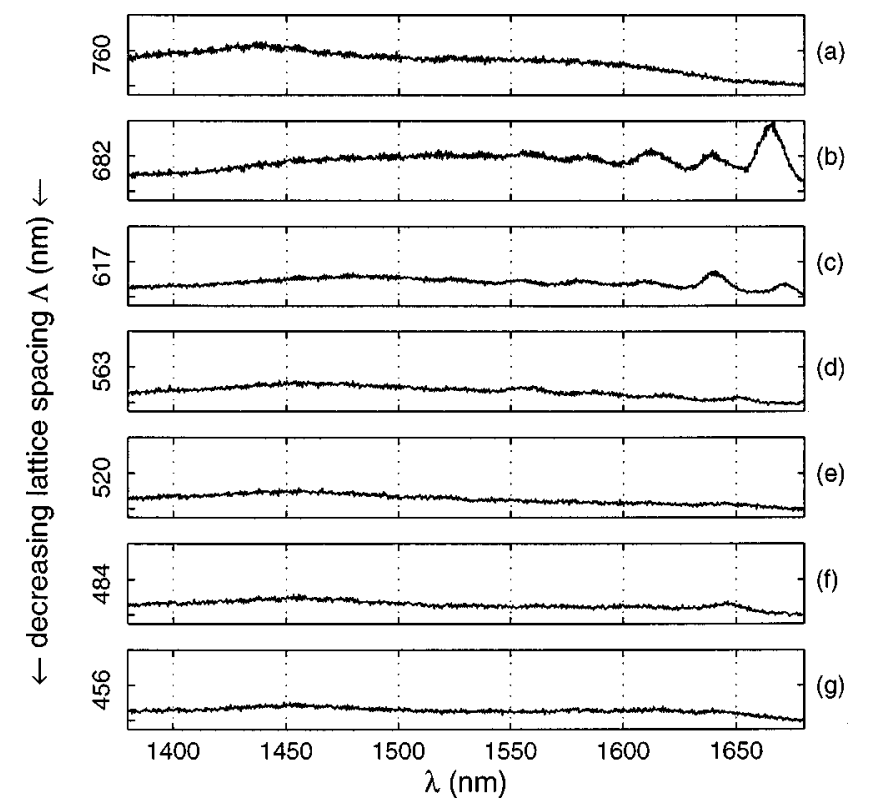

FIG. 3. Normalized vertical emission from hexagonal membrane cavities. The lattice parameter for each spectrum is shown (in $\mathrm{nm}$ ) to the left of each curve decreasing from (a) to (g). The resonances only appear for lattice parameters where the emission is tuned to the photonic band gap.

similar to measured emission from unpatterned material. Cavity resonances can be seen in Fig. 3(b) (lattice spacing $a \approx 682 \mathrm{~nm}$ ) and at $a \approx 617 \mathrm{~nm}$ [Fig. 3(c)]. For larger lattice spacings [Fig. 3(a), $a \approx 760 \mathrm{~nm}$ ] no cavity resonances are evident. For shorter lattice spacings the resonances are barely visible at $a \approx 563 \mathrm{~nm}$ [Fig. 3(d)] and then disappear for subsequent lattice sizes [Figs. 3(e)-3(g)]. This result is consistent with a cavity resonance reflecting from the photonic band gap crystal. If the reflection were a simple Fresnel reflection from the air interface or an effective index-type Fresnel reflection, the cavity resonance spacing would be seen to tune only with the cavity size (proportional to $a$ ) and resonances would be expected for all lattice sizes. The cavity resonances in Fig. 3(b) $(a \approx 682 \mathrm{~nm})$ appear with a spacing of $\Delta \lambda \approx 26.4 \mathrm{~nm}$. Using a simple Fabry-Pérot approximation and a material index of $n=3.6$, this gives an effective cavity length of $d=14.5 \mu \mathrm{m}$. This corresponds well with the hexagon size (face to face) measured by scanning electron micrograph [Fig. 1(a)] of approximately $14 \mu \mathrm{m}$. For this device with strong cavity resonances, the normalized emitted power can also be seen to be larger than for detuned band gaps (e.g., Figs. 3(a) and 3(c)). The cavity resonances in Fig. 3 are seen to be strongest near a hole spacing of $a=682 \mathrm{~nm}$ as compared to the calculated band gap center for this structure for a whole spacing of $500 \mathrm{~nm}$ as mentioned previously. However, due to strong heating in the device, the emission peak was redshifted significantly as compared to the original 1550 emission wavelength. Also because of the dramatic temperature change the material index and structure size (due to thermal expansion) are also slightly shifted which could account for the difference between the calculated design and the measured resonances.

Figures 4(a)-4(d) show variations in the cavity resonances as the photonic crystal hole size is increased with a constant lattice spacing, thereby varying $r / a$ around the deo AlP license or copyright, see http://apl.aip.org/apl/copyright.jsp 


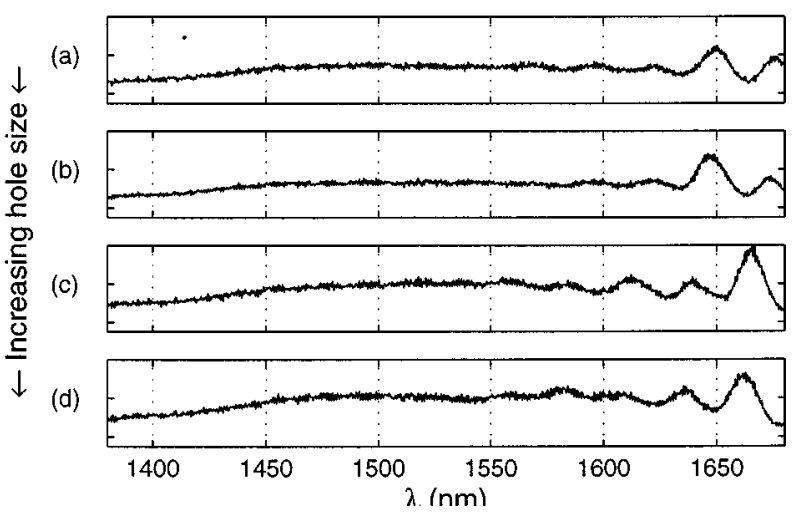

FIG. 4. The spectra are for cavities with the same nominal lattice spacing but varying the $r / a$ parameter. These devices correspond to $a \approx 682 \mathrm{~nm}$ showing the strongest resonances in Fig. 3. (c) Corresponds to Fig. 3(b).

sign value $r / a=0.31$. Figure 4(c) corresponds to the same device as shown in Fig. 3(b). As the value of $r / a$ is increased, a shift of the band gap towards higher frequencies (shorter wavelength) is expected. ${ }^{12}$ This shift can be seen in Fig. 4 where the peak at $\lambda \approx 1650 \mathrm{~nm}$ in Fig. 4(a) gradually shifts to a shorter wavelength of $\lambda \approx 1635 \mathrm{~nm}$ in Fig. 4(d). However, Fig. 4 shows that the strongest peak appears to jump to a longer wavelength from Figs. 4(b) to 4(c). These data are difficult to interpret because of the strong heating effects seen in these devices.

In summary, we have demonstrated active light emitting devices based on two-dimensional photonic band gap structures in the InP material system, emitting in the near infrared. We have shown that confinement of the spontaneous emission can be controlled, depending on the wavelength of emission and the design of the photonic band gap. Strong Fabry-Pérot-like resonances were observed which appeared only for correctly tuned photonic band gap patterns. Also, the spontaneous emission extraction efficiency was demonstrated to be significantly higher with the presence of a correctly tuned photonic band gap as compared to a mistuned or unpatterned sample. By further reducing the cavity volume and optimizing the cavity design for high $Q$, these membrane cavities show promise as highly efficient light emitters and to observe fundamental changes in the spontaneous emission properties in a semiconductor light emitting device.

The authors would like to thank the Ortel Corporation for supplying the material grown for this letter. This work was supported by the U.S. Army Research Office. R.K.L. and O.J.P. are both supported by the National Science and Engineering Research Council of Canada. The authors would like to thank Dr. J. O'Brien from the University of Southern California for helpful discussions.

${ }^{1}$ E. Yablonovitch, Phys. Rev. Lett. 58, 2059 (1987).

${ }^{2}$ S. John, Phys. Rev. Lett. 58, 2486 (1987).

${ }^{3}$ E. Schubert, N. Hunt, M. Micovic, R. Malik, D. Sivco, A. Cho, and G. Zydzik, Science 265, 943 (1994).

${ }^{4}$ S. Fan, P. Villeneuve, J. Joannopoulos, and E. Schubert, Phys. Rev. Lett. 78, 3294 (1997).

${ }^{5}$ J. O'Brien, O. Painter, R. Lee, C. Cheng, A. Yariv, and A. Scherer, Electron. Lett. 32, 2243 (1996).

${ }^{6}$ A. Rosenberg, R. Tonucci, and E. Bolden, Appl. Phys. Lett. 69, 2638 (1996).

${ }^{7}$ P. Gourley, J. Wendt, G. Vawter, T. Brennan, and B. Hammons, Appl. Phys. Lett. 64, 687 (1994).

${ }^{8}$ D. Labilloy, H. Benisty, C. Weisbuch, T. Krauss, R. D. L. Rue, V. Bardinal, R. Houdré, U. Oesterle, D. Cassagne, and C. Jouanin, Phys. Rev. Lett. 79, 4147 (1997).

${ }^{9}$ J. Martorell and N. Lawandy, Phys. Rev. Lett. 65, 1877 (1990).

${ }^{10}$ E. Petrov, V. Bogomolov, I. Kalosha, and S. Gaponenko, Phys. Rev. Lett. 81, 77 (1998).

${ }^{11}$ M. Berggren, A. Dodabalapur, R. Slusher, Z. Bao, A. Timko, and O. Nalamasu, Electron. Lett. 34, 90 (1998).

${ }^{12}$ J. Joannopoulos, R. Meade, and J. Winn, Photonic Crystals, Molding the Flow of Light (Princeton University Press, Princeton, NJ, 1995).

${ }^{13}$ O. Painter, J. Vuckovic, and A. Scherer, J. Opt. Soc. Am. B 16, 275 (1999).

${ }^{14}$ P. S. J. Russell, D. M. Atkin, and T. A. Birks, in Microcavities and Photonic Bandgaps (Kluwer, Dordrecht, 1996), pp. 203-218.

${ }^{15}$ C. Cheng, V. Arbet-Engels, E. Yablonovitch, and A. Scherer, J. Vac. Sci. Technol. B 14, 4110 (1996).

${ }^{16}$ A. Scherer, O. Painter, B. D’Urso, R. Lee, and A. Yariv, J. Vac. Sci. Technol. B 16, 3906 (1998).

${ }^{17}$ A. Levi, S. McCall, S. Pearton, and R. Logan, Electron. Lett. 29, 1666 (1993).

${ }^{18}$ Landolt-Bornstein, Numerical Data and Function Relationships in Science and Technology (Springer, Berlin, 1982), Vol. 17a-b.

${ }^{19}$ Y. Xu, J. Vuckovic, R. Lee, O. Painter, A. Scherer, and A. Yariv, J. Opt. Soc. Am. B 16, (1999). 